\title{
La Importancia del Pensamiento Filosófico y Científico en la Generación del Conocimiento ${ }^{1}$ Importance of Philosophical and Scientific
}

DOI: http://dx.doi.org/10.17981/cultedusoc.9.1.2018.05

Artículo de reflexión. Recibido: 12 de agosto de 2017 / Aceptado: 11 de diciembre de 2017

José Eriberto Cifuentes Medina² y Aura Lucia Camargo Silva ${ }^{3}$

Universidad Pedagógica y Tecnológica de Colombia (Colombia)

joseeriberto.cifuentes@uptc.edu.co

Para citar este artículo

Cifuentes, J. y Camargo, A. (2018). La Importancia del Pensamiento Filosófico y Científico en la Generación del Conocimiento. Cultura. Educación y Sociedad 9(1), 69-82. DOI: http://dx.doi.org/10.17981/cultedusoc.9.1.2018.05

\section{Resumen}

El objeto de la disertación, es concertar que el pensamiento filosófico y el científico han contribuido en la generación de conocimiento a lo largo de la historia y se consolida en el siglo XX y ahora la incidencia en el XXI. La filosofía es una actividad mental por medio de la cual construimos el laberinto armónico de las ideas, muchas de ellas encargadas de vivencias y algunas tendientes a la práctica. Para Wittgenstein, la filosofía no es una ciencia sino una actividad, razón por la cual la palabra filosofar, connota un sentido dinámico, de deconstrucción, de re pensamiento, de construcción. Para Radnitzky, el papel de la filosofía: es construir sinopsis de sistemas de conocimientos producidos por la ciencia; así la filosofía es una metaciencia. Razón por la cual el pensamiento filosófico conlleva a la reflexión e induce a la generación de conocimiento en cada época de la historia y se ha fortificado en los últimos siglos, especialmente en el siglo XX, en donde se ha abordado: la Filosofía de la Ciencia, que, desde diversas miradas, la epistemología ha adquirido un fundamento significativo desde sus argumentos. Por los siglos y en la actualidad, la filosofía se ha preguntado e intentado dar respuestas racionales a cuestiones como: ¿Qué?, ¿Por qué?, ¿Quién?, ¿Para qué? Y la pregunta clave de la filosofía es el ¿Por qué? Inquietud esencial y que hace vital a la filosofía al menos la filosofía griega dedicada a la búsqueda del "Principio" de las cosas tanto a nivel de la realidad como a nivel del conocimiento. De manera que, si no se ha de ver en la filosofía algo de admiración, es necesario atacarla, quizás destruirla para que finalmente caiga en el olvido.

Palabra Clave: Pensamiento Filosófico, Científico, Generación de Conocimiento

\begin{abstract}
The object of the dissertation is to conclude that the philosophical and scientific thought have contributed to the generation of knowledge throughout history and is consolidated in the twentieth century and now the incidence in the XXI. Philosophy is a mental activity through which we build the harmonic labyrinth of ideas, many of them charged with experiences and some aimed at practice. For Wittgenstein, philosophy is not a science but an activity, why philosophize word connotes a dynamic sense, deconstruction, re thinking construction. To Radnitzky, the role of philosophy is to build systems synopsis of knowledge produced by science; so philosophy is a metascience. Why philosophical thought leads to reflection and leads to the generation of knowledge in every period of history and has been fortified in recent centuries, especially in the twentieth century, which has been addressed: The Philosophy of Science which, from different looks, epistemology has gained significant ground since its arguments. Forever and today, the philosophy has been asked and tried to give rational answers to questions such as: What? Why? Who? Why? And the key question of philosophy is the Why? essential concern and that makes it vital to philosophy at least Greek philosophy dedicated to the pursuit of the "principle" of things both at the level of reality and at the level of knowledge. So, if you have not seen in some admiration philosophy, it is necessary to attack, destroy perhaps to finally fall into oblivion.
\end{abstract}

Keywords: Philosophical, Scientific, Knowledge Generation

\footnotetext{
${ }^{1}$ El artículo hace parte del proyecto titulado: Las tendencias pedagógicas en el proceso de enseñanza y formación socio humanística y ética en estudiantes de una licenciatura en educación básica.

${ }^{2}$ Doctorando y Magister en Educación. Especialista en Educación con énfasis en Evaluación Educativa. Licenciado en Teología. Licenciado en Filosofía y Educación religiosa de la Universidad Santo Tomas. Docente Universidad Pedagógica y Tecnológica de Colombia.

${ }^{3}$ Magister en Educación y Licenciada en Matemáticas y Física de la Universidad Pedagógica y Tecnológica de Colombia. Docente Universidad Pedagógica y Tecnológica de Colombia.
} 


\section{Introducción}

A lo largo de los siglos la Filosofía como sabiduría total ha ido perdiendo espacio por múltiples circunstancias, terreno que pretendemos recuperar ya al llegar al siglo XXI; preguntar y preguntarse, he ahí la llave de entrada a la Filosofía: Es la puerta que nos introduce a la reflexión y a la posterior respuesta. Lo problémico suscita la inquietud problematizadora a nivel mental y la consiguiente reflexión bajo la vivencia de la verdad, de la pregunta o pensamiento filosófico nacen la investigación científica o pensamiento científico y los dos confluyen en la reflexión y generación valida de conocimiento.

La razón de ser de la presente disertación, está en la búsqueda de los afluentes que conducen al rio de la afirmación: "el pensamiento filosófico y científico han contribuido en la generación de conocimiento a lo largo de la historia y se consolida en el siglo XX y XXI". Las diversas fuentes han de conducir a fortalecer o desvirtuar el problema planteado, desde diversas miradas de intelectuales y corrientes de pensamiento. La preocupación de intelectuales, filósofos y científicos es la búsqueda de una autenticidad en la filosofía de estas latitudes es el "ontos" del conocimiento generado en la controversia científica y de la reflexión filosofía.

El hombre se formula interrogantes más a menudo, referentes al problema del conocimiento: ¿Es posible conocer?, ¿Cuál es el origen del conocimiento? ¿Cuál es la esencial del conocimiento?, ¿Cuántas y cuales formas de conocimiento hay?, ¿Cuáles son los criterios de validez y de verdad del conocimiento? ¿Qué hace que a un conocimiento se le denomine conocimiento científico? La Filosofía y la Ciencia desde sus disciplinas y corrientes que les asisten en apoyo, a partir sus reflexiones y expectantes de sus postulados; promueven la comprensión de interpretación y generan la validación del conocimiento filosófico y científico.

La disertación con apoyo de AsensiArtiga y Parra-Pujante (2002), Valdés (2002), Bojaca (2000), Suarez (2003), Gandía (2004), Azcárraga (2002), Rivadulla (2004), Iranzo (2005) entre otros y se organiza en varios apartados, de manera que se consolida la validación de la afirmación en relación al acercamiento a la filosofía de la ciencia, hacia una nueva Filosofía de la Ciencia, el método científico como camino para validar el conocimiento resultado de la reflexión filosófica y las tendencias epistemologías del siglo XX y su proyección en el siglo XXI.

\section{Acercamiento a la Filosofía de la Ciencia}

El artículo discute acerca del engranaje entre el pensamiento filosófico y científico, en las razones posibles de cómo han contribuido en la generación de conocimiento, En la perspectiva de Iranzo (2005), afirma que;

Kuhn no fue el primero en reclamar un papel crucial para la historia en la filosofía de la ciencia (Duhem y Whewell serían dos ejemplos destacados del siglo XIX), su consigna historicista es un reto al modo en que los empiristas lógicos y sus discípulos entendieron la filosofía de la ciencia. 
Kuhn pretendía el acercamiento a la ciencia "real", tal como efectivamente ha sido practicada, $y$ se posicionaba en contra de los enfoques formalistas derivados del empirismo lógico -la "Concepción Heredada"

En el seno de la Filosofía y la Ciencia, como posibilidad de fuente y generación de conocimiento a lo largo de la historia y su acentuada connotación desde el siglo XX y XXI. La relación entre el filósofo y en científico, se puede inferir es cercana dadas las circunstancias de implicación epistemológica y ontológica de correlación, que en apoyo de otras ciencias y disciplinas fortalecen el principio de generación de conocimiento.

Se puede evidenciar que la Filosofía es una ciencia pero que se puede hacer Filosofía de la ciencia y por ello el filosofar es pensar y/o reflexionar filosóficamente sobre algo, que para el caso es: La Ciencia. Entre las dos está de emparentada marcación el conocimiento, como eje central de análisis. La Filosofía se consolida como fuente y generación de conocimiento entre la que considera que se da bajo la observación y se aduce que se requiere de una estructura racional. Surge entonces, la siguiente pregunta:

¿Cuál es el papel de la filosofía en los inicios del Siglo XXI y del tercer milenio?" y a renglón seguido responde: Se le asigna a la filosofía el papel de construir sinopsis de sistemas de conocimientos producidos por la ciencia; así la filosofía es una metaciencia. La metafilosofía es el análisis de la actividad filosófica por medio del lenguaje filosófico. (Bojacá, 2000)
La filosofía de la ciencia es el estudio de los supuestos, fundamentos y conclusiones de la ciencia, investiga las diferentes ramas de la ciencia y su estructura subyacente. Las preguntas centrales son: “QQué es la ciencia?” y "¿Qué no es ciencia?", así como también "¿Qué caracteriza a la ciencia?” y "¿Cómo lograr el progreso científico?”. La filosofía de la ciencia intenta distinguir la ciencia de la religión y la pseudociencia. El cisma entre la ciencia y la religión comenzó en el siglo XVII. Fue una etapa necesaria en el avance del conocimiento humano, en la medida que genera la reflexión filosófica y científica se va consolidando la generación de nuevo conocimiento, validado por el método.

A lo largo de la historia se han dado muchos giros al concepto de Filosofía, sin embargo, cobra especial importancia y se remarca la concepción de los primeros filósofos griegos; quienes optaron por llamarse amigos de la sabiduría, estudiosos y aficionados con la conciencia socrática del: Solo sé que nada se. El este sentido, Gandía (2004) afirma que:

Tradicionalmente la filosofía de la ciencia se adscribe en la corriente de la filosofía analítica, con lo que estaba centrada principalmente en un análisis de los lenguajes utilizados en las prácticas científicas. La filosofía de la ciencia se hallaba alineada, pues, dentro de la hegemónica corriente del positivismo científico, liderado intelectualmente en aquellos años por el Círculo de Viena (p. 1).

Para la filosofía de la ciencia tradicional hay una sola realidad dada de una vez por siempre para todos los humanos. Una realidad que puede ser observada y teorizada. La filosofía de la ciencia tradicional: 
Persigue lo que debe ser, frente al planteamiento de Kuhn y Feyerabend que se orienta al análisis del ser. No es sólo una metodología de la ciencia, sino sobre todo un análisis del lenguaje de las teorías científicas. Desde esta visión clásica la filosofía de la ciencia es una reconstrucción racional y sistemática de las teorías científicas. No es sólo una metodología de la ciencia, sino sobre todo un análisis del lenguaje de las teorías científicas. Desde esta visión clásica la filosofía de la ciencia es una reconstrucción racional y sistemática de las teorías científicas (Gandía, 2004, p. 13).

La Filosofía y la Ciencia, en los tiempos actuales se coadyuvan en la validación y generación de nuevo conocimiento, El este sentido, Azcárraga (2002) afirma que:

La filosofía no puede, hoy, vivir de espaldas al conocimiento científico, y menos aún, tratar de relativizarlo en un intento fútil de retener un espacio privilegiado o protegido, que es lo que, en el fondo, han intentado algunos filósofos postmodernos con sus críticas desmesuradas a la racionalidad y objetividad de la ciencia. La filosofía debe estar, hoy, unida a la ciencia. La ciencia nos ayuda a comprender cómo son las cosas, no cómo nos parecen o desearíamos que fueran, y por tanto es, a largo plazo, más inmune a nuestras preconcepciones, filias y fobias (p. 2).

A mediados del siglo XX, tres filósofos de la ciencia presentaron, sus aportes acerca del pensamiento científico como estos planteamientos validados permiten la convergencia de la generación del conocimiento (Tabla 1).
Tabla 1.

La división de la filosofía en el siglo XX

\begin{tabular}{|c|c|}
\hline Filosofo & Breve Descripción \\
\hline $\begin{array}{c}\text { Karl } \\
\text { Popper }\end{array}$ & $\begin{array}{l}\text { El conocimiento científico es } \\
\text { progresivo y acumulativo, pero } \\
\text { "falsable", con lo que únicamente } \\
\text { se puede considerar ciencia lo } \\
\text { que puede ser cuestionado }\end{array}$ \\
\hline $\begin{array}{l}\text { Thomas } \\
\text { Kuhn }\end{array}$ & $\begin{array}{l}\text { El conocimiento científico no es } \\
\text { necesariamente progresivo, sino } \\
\text { una respuesta a las demandas } \\
\text { sociales, y en la mayor parte de } \\
\text { los casos, la "ciencia normal" } \\
\text { es únicamente el constante } \\
\text { esfuerzo por confirmar el vigente } \\
\text { paradigma, que únicamente } \\
\text { cambiará por una revolución } \\
\text { científica, de las que ha habido } \\
\text { muy pocas históricamente }\end{array}$ \\
\hline $\begin{array}{c}\text { Paul } \\
\text { Feyerabend }\end{array}$ & $\begin{array}{l}\text { El conocimiento científico no } \\
\text { es acumulativo o progresivo, } \\
\text { sino inconsistente y anárquico } \\
\text {-anarquismo epistemológico-, } \\
\text { no habiendo criterio de } \\
\text { demarcación, en términos de } \\
\text { método, entre lo que suele } \\
\text { llamarse "ciencia" y cualquier } \\
\text { otra forma de investigación }\end{array}$ \\
\hline
\end{tabular}

Fuente: Elaboración Propia

Especialmente desde la publicación y divulgación de los libros de Popper (La lógica de la investigación científica, 1934 y 1959), Kuhn (La estructura de las revoluciones científicas, 1962) y Feyerabend (Contra el método, 1975), se han generado constantes debates en las comunidades científicas y académicas, tanto en el ámbito de las llamadas: ciencias duras como el de las: ciencias blandas, el de las ciencias físico-naturales y el de las humanidades y ciencias sociales (o humanas, o ciencias morales y políticas), sobre la naturaleza, significado, objetividad, subjetividad, capacidad analítica, sintética y predictiva de la ciencia; el cuestionamiento del objeto y la metodología propios de cada ciencia, las ventajas e inconvenientes de 
la especialización y el reduccionismo, las posibilidades de interdisciplinariedad y de perspectivas holísticas; y la relación del conocimiento científico con los conceptos de verdad y de realidad.

Por ello, y rememorando el universalismo del renacimiento, la filosofía del s. XXI deberá incluir en su bagaje el conocimiento científico o no será filosofía, es decir, amor a la sabiduría. Además, se ha de observar que la filosofía de la ciencia puede describir cómo se lleva a cabo la investigación y un modo de decidir la forma en que debe llevarse a cabo, y abordar el siguiente interrogante: ¿cómo se relacionan entre sí el mundo real, la información empírica, los modelos y las teorías y qué se puede hacer para mejorar su relación?

\section{Hacia una nueva Filosofía de la ciencia}

En la perspectiva de Iranzo (2005) que citando a Rossi (1990), refiere la dinámica cambiante entre la Ciencia y la Filosofía. En el propósito de sostener la hipótesis de la existencia de una nueva filosofía de la ciencia, según afirma Rossi (1990), en los siguientes términos:

... para entender el surgimiento de las nuevas ciencias y los cambios profundos que acompañan el crecimiento de las ciencias de más antigua tradición... es necesario, por ejemplo, analizar el significado de la transición entre diferentes imágenes de la ciencia o diferentes imágenes del sabio, del docto, del científico. Sólo a través de ese análisis es posible, en efecto, determinar los fines asignados a la ciencia en una época determinada, las reglas que se deben respetar en ella. Determinar esos fines y esas reglas quiere también decir indicar los criterios considerados aceptables para optar entre hipótesis y entre teorías.
En aras de avanzar en la disertación de la Filosofía de la ciencia y la nueva filosofía de la ciencia en apoyo del método científico, como elemento que contribuye a la validación del nuevo conocimiento; se pretende en primer lugar hacer un trazado general y sistemático de la historia de la Ciencia, con el propósito de acceder a los postulados de Asensi-Artiga y ParraPujante (2002), quienes afirman que su artículo:

No pretende ser un esbozo de filo-
sofía de la ciencia, sino algo más
modesto: un rápido repaso a los
caminos de ésta a lo largo del siglo
XX recién acabado con el fin de
mostrar, tras el giro lingüístico
que la filosofía dio a comienzos
de esa centuria con autores como
Rusell o el llamado 'primer Witt-
genstein', el autor del Tractatus
Logico- Philosophicus ciertos ja-
lones de la nueva filosofía de la
ciencia, hasta llegar a la orienta-
ción que podríamos llamar his-
toricista, para cuyos defensores
más extremos, como Feyerabend,
habría que proceder a una especie
de desconstrucción que despejara
las verdadera razones de uno u
otro paradigma (p. 12).

Antes de abordar la ocupación de Asensi-Artiga y Parra-Pujante (2002), se acude de manera general y resumida a la historia de la filosofía como antecedente y consecuente de la nueva filosofía de la ciencia. La primera tiene sus raíces en la filosofía griega y surgió como una disciplina autónoma en el siglo XIX. Aunque ya a partir de antes, filósofos e investigadores se interesaron en la Filosofía de la Ciencia, desde épocas remotas (Tabla 2). 
Tabla 2.

Breve historia de la filosofía de la ciencia Siglo XV, XVI y XVII

\begin{tabular}{|c|c|}
\hline Autor & Planteamiento \\
\hline $\begin{array}{l}\text { Nicolás Copérnico } \\
\text { (1473 - 1543,Polonia) }\end{array}$ & $\begin{array}{l}\text { Fue un astrónomo del Renacimiento que formuló la teoría } \\
\text { heliocéntrica del Sistema Solar }\end{array}$ \\
\hline $\begin{array}{c}\text { Galileo Galilei } \\
\text { (1564- 1642, Italia) }\end{array}$ & $\begin{array}{l}\text { Sus logros incluyen la mejora del telescopio, gran variedad de } \\
\text { observaciones astronómicas, la primera ley del movimiento } \\
\text { y un apoyo determinante a la teoría de Copérnico. }\end{array}$ \\
\hline $\begin{array}{c}\text { Francis Bacon } \\
(1561-1626, \text { Inglaterra })\end{array}$ & $\begin{array}{l}\text { Es considerado uno de los padres del empirismo, sus obras } \\
\text { y pensamientos ejercieron una influencia decisiva en el } \\
\text { desarrollo del método científico. }\end{array}$ \\
\hline $\begin{array}{c}\text { Johannes Kepler } \\
\text { (1571 -1630, Alemania) }\end{array}$ & $\begin{array}{l}\text { Conocido fundamentalmente por sus leyes sobre el } \\
\text { movimiento de los planetas en su órbita alrededor del Sol. }\end{array}$ \\
\hline $\begin{array}{c}\text { Isaac Newton } \\
\text { (1643-1727, Inglaterra) }\end{array}$ & $\begin{array}{l}\text { La ley de la gravitación universal y estableció las bases de la } \\
\text { mecánica clásica mediante las leyes que llevan su nombre, } \\
\text { también se destacan sus trabajos sobre la naturaleza de la } \\
\text { luz y la óptica y el desarrollo del cálculo matemático. }\end{array}$ \\
\hline $\begin{array}{l}\text { Albert Einstein } \\
\text { (1879- 1955, Alemania) }\end{array}$ & $\begin{array}{l}\text { Presentó la teoría de la relatividad general, en la que } \\
\text { reformuló por completo el concepto de gravedad. Estudio } \\
\text { científico del origen y la evolución del universo por la rama } \\
\text { de la física denominada cosmología. }\end{array}$ \\
\hline
\end{tabular}

Fuente: Elaboración propia, argumentada en Valdés (2015).

Tabla 3.

Breve historia de la filosofía de la ciencia Siglo XVIII

\begin{tabular}{|c|c|}
\hline Autor & Planteamiento \\
\hline $\begin{array}{c}\text { Augusto Comte } \\
\text { (1798 -1857, Francia) }\end{array}$ & $\begin{array}{l}\text { Creó la palabra altruismo (dar sin esperar nada a cambio) su } \\
\text { filosofía tuvo gran repercusión que se fundaron países como } \\
\text { Brasil en función de su filosofía, país en cuya bandera se } \\
\text { lee Orden y Progreso, parte de la triada filosófica de Comte } \\
\text { (Altruismo, Orden, Progreso). Miraba a la ciencia como } \\
\text { una fuente de progreso, la forma en la que organizamos las } \\
\text { universidades, etc. }\end{array}$ \\
\hline $\begin{array}{c}\text { John Stuart Mill } \\
\text { (1806 -1873, Inglaterra) }\end{array}$ & $\begin{array}{l}\text { Fue un seguidor del positivismo de Comte, también fue } \\
\text { representante del pensamiento romántico y del socialismo. } \\
\text { Planteó que el conocimiento científico es meramente } \\
\text { probable, no necesario. } \\
\text { Numerosos científicos, sin embargo, se han dado por } \\
\text { satisfechos dejando la filosofía de la ciencia a los filósofos } \\
\text { y han preferido seguir haciendo ciencia en vez de dedicar } \\
\text { más tiempo a considerar cómo se hace la ciencia. El debate } \\
\text { realismo - anti realismo es un debate filosófico que explora los } \\
\text { fundamentos de la verdad científica comúnmente aceptada. }\end{array}$ \\
\hline $\begin{array}{c}\text { David Hume } \\
\text { (1711 - } 1776, \text { Inglaterra })\end{array}$ & $\begin{array}{l}\text { Por su parte el reduccionismo científico es una idea muy } \\
\text { debatida en la filosofía de la ciencia, donde la ciencia reduce } \\
\text { las interacciones y entidades complejas a la suma de las } \\
\text { partes que la componen. }\end{array}$ \\
\hline
\end{tabular}

Fuente: Elaboración propia, argumentada en Valdés (2015). 
En el siglo XVIII, la filosofía de la ciencia ya se es considerada como una disciplina autónoma y puede ser vista como una manera de describir cómo se lleva a cabo la investigación y estudia ¿cómo se relacionan entre sí el mundo real, la información empírica, los modelos y las teorías y qué se puede hacer para mejorar su relación. Con Augusto Comte cambian los paradigmas de la filosofía y de la ciencia y a renglón seguido otros filósofos y científicos realizan sus planteamientos (Tabla 3).

Numerosos científicos, sin embargo, se han dado por satisfechos dejando la filosofía de la ciencia a los filósofos y han preferido seguir haciendo ciencia en vez de dedicar más tiempo a considerar cómo se hace la ciencia. Según Valdés (2015) y los postulados de Asensi-Artiga y Parra-Pujante (2002), se puede considerar, que la Filosofía de la Ciencia alcanza su edad adulta en los años de 1920 con la aparición del Círculo de Viena, integrado por un grupo de filósofos como: Rudolf Carnap (1891-1970), Otto Neurath (1881-1945), Hans Hahn (1879-1934), Kurt Gödel (1906-1978), Willard V. Quine (19082000).

El Círculo de Viena encabezado por el Dr. Johan Craidoff (1899 - 1960, Alemania) propuso un modelo de ciencia en el que ésta procede mediante generalizaciones (inducción) a partir de los datos. Propuso la idea central del positivismo y del neopositivismo donde la ciencia debe utilizar las teorías como instrumentos para predecir fenómenos observables y debe renunciar a buscar explicaciones. La búsqueda de explicaciones es función de la metafísica, que no es ciencia sino palabrería carente de significado.
Con el progreso de la ciencia ésta comenzó el estudio de campos que están más allá de la experiencia, como puede ser la física de altas energías o la física atómica. En esta situación el criterio empirista de verdad condujo a muchos problemas, lo que llevó a diversas matizaciones del mismo. El verificacionismo estricto, acabó siendo abandonado y sustituido por la contrastación entre proposiciones y observaciones, lo que permite una confirmación gradualmente creciente de las teorías.

Asensi-Artiga y Parra-Pujante (2002), proponen además que se hace necesario "iniciar a los estudiantes en el conocimiento de la investigación científica, es necesario introducirlos en el estudio de la naturaleza de la ciencia y de todos aquellos elementos que permiten el desarrollo de sus objetivos" (p. 12); evocando a autores como Sierra, Bunge y Kohan que proponen, las siguientes definiciones de Ciencia (Tabla 4).

Tabla 4.

Definiciones de Ciencia

\begin{tabular}{cl}
\hline Autor & \multicolumn{1}{c}{ Definición } \\
\hline \multirow{3}{*}{ Sierra } & $\begin{array}{l}\text { Define la ciencia en sentido estricto, } \\
\text { como un conjunto sistemático de co- } \\
\text { nocimientos sobre la realidad obser- } \\
\text { vable, obtenidos mediante el método } \\
\text { de investigación científico. }\end{array}$ \\
& $\begin{array}{l}\text { Indica que conviene delimitar el con- } \\
\text { cepto de Tecnología, que para él con- } \\
\text { Bunge } \\
\text { siste en el desarrollo de la actividad } \\
\text { científica aplicada al mejoramiento de } \\
\text { nuestro medio natural y artificial, a } \\
\text { la invención y manufactura de bienes } \\
\text { materiales y culturales. } \\
\text { El objetivo principal de una ciencia, } \\
\text { más que una mera descripción de } \\
\text { fenómenos empíricos, es establecer, } \\
\text { mediante leyes y teorías, los prin- } \\
\text { cipios generales con que se pueden } \\
\text { explicar y pronosticar los fenómenos } \\
\text { empíricos. }\end{array}$ \\
\hline
\end{tabular}

Fuente: Asensi-Artiga y Parra-Pujante (2002) 
En el seguimiento sistemático de Asensi-Artiga y Parra-Pujante (2002) y Valdés (2002), permite confirmar que hay un proceso histórico y evolutivo de la Filosofía de la Ciencia que para el caso se intenta rastrear desde el siglo XV, llegando a convertirse en una disciplina autónoma en el siglo XVIII, avanzando su consolidación en el siglo XIX y en el siglo $\mathrm{XX}$, que ha llegado a un proceso de maduración con el Circulo de Viena e intelectuales como Popper y Kuhn; la impulsan hacia una nueva Filosofía de la Ciencia

De la filosofía tradicional según los planteamientos de Gandía (2004) a la nueva filosofía en los razonamientos de Asensi-Artiga y Parra-Pujante (2002), permiten inferir que:
Los caminos de la filosofía de la ciencia a lo largo del siglo XX, tras el giro lingüístico que la filosofía dio en los primeros años de esta centuria, llegan hasta la orientación historicista de la ciencia, para cuyos defensores más extremos habría que proceder a una desconstrucción que despejara las verdaderas razones de uno u otro paradigma. El método científico y la capacidad de la razón nos permiten seguir avanzando en busca de la verdad y la creación de modelos para aumentar nuestro conocimiento científico del mundo (p. 9).

Se puede constatar que, las mayores contribuciones a la filosofía de la ciencia durante el siglo XX han surgido, sin duda, en el seno de esta tradición filosófica (Carman, 2007). De acuerdo con Carman (2007), la filosofía de la ciencia de corte analítico del siglo XX puede dividirse en tres etapas (Tabla 5).

Tabla 5.

La división de la filosofía en el siglo XX

\begin{tabular}{|c|c|}
\hline $\begin{array}{l}\text { 1. Clásico o concepción } \\
\text { heredada }\end{array}$ & $\begin{array}{l}\text { Para este primer período, las teorías científicas son } \\
\text { consideradas sistemas axiomáticos empíricamente } \\
\text { interpretados. Se destaca, fundamentalmente, el } \\
\text { análisis sincrónico de las teorías, es decir, el estudio } \\
\text { de la estructura de una teoría abstraída de su devenir } \\
\text { en el tiempo. En esta parte veremos al Círculo de Viena } \\
\text { en general y a Carnap y Hempel más en particular. }\end{array}$ \\
\hline 2. Historicista & $\begin{array}{l}\text { En esta etapa hay un interés cada vez más marcado } \\
\text { por la historia de la ciencia, tratando de confrontar } \\
\text { con ella las propuestas metodológicas. Se caracteriza } \\
\text { por propuestas diacrónicas. Las teorías científicas son } \\
\text { vistas como "proyectos de investigación". Se destacan } \\
\text { Kuhn, Lakatos, Feyerabend, Laudan, Hanson, } \\
\text { Toulmin. }\end{array}$ \\
\hline $\begin{array}{l}\text { 3. Contemporáneo o } \\
\text { semántico }\end{array}$ & $\begin{array}{l}\text { Que va desde mediados de los ' } 80 \text { hasta nuestros } \\
\text { días. Se destacan actualmente Ian Hacking, Nancy } \\
\text { Cartwright, Bas Van Fraassen, Richard Boyd, Rom } \\
\text { Harré, Joseph Sneed y otros. Para la mayoría de } \\
\text { estos autores, las teorías son mejor caracterizadas } \\
\text { como entidades modelo-teóricas. Aquí veremos dos } \\
\text { concepciones semánticas de la ciencia pero muy } \\
\text { distintas: la de la concepción estructuralista y la de } \\
\text { Rom Harré. }\end{array}$ \\
\hline
\end{tabular}

Fuente: Elaboración propia, argumentada en: Carman (2007). 
La idea de tolerancia metodológica es muy importante, porque muestra a un Lakatos mucho más flexible frente a lo excesivamente estricto que es Popper. Además, Lakatos no comparte con Popper la ilusión de que una mejor filosofía de la ciencia ayude a la ciencia, la metodología ayuda al histórico de la ciencia, pero no al científico (Carman, 2007). En el siglo XX, en el Círculo de Viena que se constituyó formalmente en 1922, en torno a la cátedra de filosofía de las ciencias inductivas que había pasado a ocupar Moritz Schlick (la misma que había sido creada para Enrst Mach en la Universidad de Viena), A partir de 1929, entonces, adquirió consistencia como una escuela con concepciones propias sobre la ciencia.

\section{El método científico, camino a la validación del conocimiento}

A lo largo de la historia, el hombre se ha enfrentado a un sinnúmero de obstáculos y problemas para desentrañar los secretos de la naturaleza, tanto para vivir con ella, como de ella en armonía. Para superar esos problemas ha empleado muy diversas estrategias, las cuales dieron paso a la formalización de procedimientos que, en última instancia, no son sino el propio método científico. Por ello en la línea de Asensi-Artiga y Parra-Pujante (2002), Bojacá (2000), Suarez (2003) y Ortiz \& García (2010), se diserta en connotación a la definición y consecuencia la relación con Filosofía de la Ciencia. En el proceso de construcción de la ciencia, de manera elemental han operado dos métodos antagónicos pero complementarios; el método inductivo de lo particular a lo general y el método deductivo de lo general a lo particular.
Por tanto, "Bojacá (2000) plantea una definición etimológica, de método: (meta odos = a lo largo del camino) se el conjunto armónico de medios para lograr un fin, en este caso para logar la ciencia; existen métodos para aprender ciencia (didáctica), métodos para trasmitir ciencia (pedagogía); métodos para crear ciencia (método científico)" (Cifuentes, p. 68). Por consiguiente, Asensi-Artiga y Parra-Pujante (2002), coincide en que, el método científico, rige toda la actividad científica, desde la gestación del problema hasta la difusión del resultado.

Asimismo, Asensi-Artiga y Parra-Pujante (2002), argumenta que: "el método científico tiende a reunir una serie de características que permiten la obtención de nuevo conocimiento científico. Es el único procedimiento que no pretende obtener resultados definitivos y que se extiende a todos los campos del saber" (p. 13). El método científico es imprescindible incluso para la superación de los mínimos exigidos para que un trabajo de investigación sea aceptado por la comunidad científica. El método científico, por lo tanto, se refiere a la serie de etapas que hay que recorrer para obtener un conocimiento válido desde el punto de vista científico, utilizando para esto instrumentos que resulten fiables. Lo que hace este método es minimizar la influencia de la subjetividad del científico en su trabajo.

Por lo tanto, Carman (2007) plantea que, nuevamente imitando el método científico, la filosofía debe proponerse un trabajo más concreto y delimitado y no que cada filósofo pretenda construir su propio sistema (como, según Carnap, hacen los metafísicos tradicionales). Finalmente, Carnap señala que mientras la nueva filosofía está en consonancia con los tiempos modernos, la metafísica tradicional es cosa del pasado. 
Los pensadores de la corriente del positivismo lógico que se da a comienzos del siglo XX, como M. Schick (1882-1936), R. Carnap (1891-1970) y H. Reichenbach (1891-1953), consideraban que la misión de la filosofía era servir como base al conocimiento a partir de la identificación de reglas que permitan transformar los datos sensibles en enunciados con valor de verdad (Verano, 2004). Dichas reglas tenían como fundamento el cálculo lógico centrado en la sintaxis del lenguaje y la importancia de la validación del conocimiento desde el pensamiento filosófico y científico, el método es el camino de la confrontación y admisión del conocimiento.

La filosofía analítica, Verano (2004), puede afirmar que según sus representantes G. E. Moore (1873-1958), L. Wittgwnstein (1899-1954), B. Russell (1872-1979) y A. J. Ayer (1900-1989), entre otros plantearon que la filosofía no puede dedicarse a otra cosa que el análisis del lenguaje. Sostuvieron que, incluso en la historia de la filosofía, el tema del lenguaje ha sido el eje central de las reflexiones.

Se puede comprobar que el surgimiento de las teorías no euclidianas con solides en sus planteamientos en el siglo XIX y soporte científico si influenciaron en la filosofía de la ciencia del siglo XX. Entre la diversidad de los filósofos están los llamados analíticos del lenguaje, que partieron de la importancia del lenguaje, con el fin de hacer una investigación más precisa y proponer la formulación de ciertas reglas, de carácter científico, cuyo uso pudiera darle un sentido verificable a los enunciados o afirmaciones que se hagan sobre la realidad.

En este sentido, Verano (2004, p. 169), afirma que "la filosofía analítica trata de determinar que el lenguaje utilizado por la filosofía en realidad tenga sentido $\mathrm{y}$, de este modo, establecer los campos o universo de cosas sobre los que se puede hablar con criterio de verdad" (En: Cifuentes \& Pedraza, 2016, p. 217). Los analistas del lenguaje como Russell, Wittgenstein, consideraron que los principios de la matemática eran los llamados a garantizar un uso lógico del lenguaje. Por ello, se les ha criticado el hecho de reducir la lógica a la matemática.

De acuerdo con el enfoque pragmático de la lógica, "el análisis del lenguaje no debe estar orientado a la creación de un lenguaje científico universal que sirva de base a las investigaciones adelantadas por las ciencias. El análisis del lenguaje, en otras palabras, no está ya al servicio de la ciencia pero que esta como base para la creación del lenguaje científico" (Verano, 2004, p. 169. En: Cifuentes \& Pedraza, 2016, p. 217).

En palabras de Senior (2001), afirma que fue extraordinario el despliegue del conocimiento matemático en el siglo XIX a través de la inventiva y la genialidad de hombres como Forurier, Gauss, Hamilton, Grassmann, Peirce entre otros muchos; en este proceso el concepto clave fue rigor. En la búsqueda a la solución de los problemas matemáticos y a las exploraciones de las ciencias empíricas llevaron a reconstruir y replantear bases para alcanzar la rigurosidad.

Entre tanto, "Senior (2001) argumenta que la idea formalista se extendió en la filosofía de la ciencia hasta abarcar las teorías de las ciencias empíricas o fácticas, especialmente con el Circulo de Viena y demás grupos, que asumieron el positivismo lógico y la visión instrumentalista" (En: Medina \& Suaréz, 2017, p. 217). Dicha arquitectura permite la construcción de conocimiento derivado de las teorías que se han consolidado en el siglo XX, derivados del profundo análisis de la teoría no euclidianas. 
La relación entre matemáticos, filósofos y lógicos conlleva a una controversia argumentada, fundante de la cadena de relación que con el rigor necesario permite plantear los fundamentos de una teoría que transciende en el tiempo; que puede desvanecer una teoría, fortalecer a otra o formular una diferente con el rigor científico necesario.

Según los argumentos de Iranzo (2005), en relación al método científico que en términos más generales se refiere a

...una concepción del método científico que descarte como no científicas prácticas corrientes, mayoritariamente aceptadas, en vastas áreas de la ciencia es cuestionable. Si tiene algún sentido distinguir entre prescripciones correctas e incorrectas, es porque el análisis guarda relación con los procedimientos y usos que efectivamente encontramos en la ciencia; y si al aplicar una propuesta prescriptiva a la práctica real nos vemos obligados a desembarazarnos de grandes porciones de ésta porque no encajan en nuestras definiciones, estamos ante una concepción idealizada o reduccionista de la ciencia, tal vez deudora de ciertos supuestos filosóficos, pero de la que no podemos decir que aporte comprensión sobre eso que en nuestro mundo llamamos ciencia.

Se puede estar interesados en explicitar las pautas normativas aplicadas en un determinado momento histórico, en un área particular de la ciencia, como la filosofía de la ciencia o las defendidas por una figura relevante en la historia de la ciencia, o más en general, por los avatares sufridos por el denominado "método científico, cuyo papel se ha considerado necesario para validar las nuevas ciencias y este caso la nueva Filosofía de la Ciencia.
Tendencias epistemológicas recientes y la generación de conocimiento

La Filosofía de la Ciencia no se denominó así hasta la formación del Círculo de Viena $^{4}$, a principios del siglo XX. En la misma época, la ciencia vivió una gran transformación a raíz de la teoría de la relatividad y de la mecánica cuántica. Entre los filósofos de la ciencia más conocidos del siglo XX figuran Karl R. Popper $^{5}$ y Thomas Kuhn, Paul Feyerabend $^{6}$, Imre Lakatos ${ }^{7}$, Ilya Prigogine, entre otros. A partir de la obra del norteamericano Thomas Kuhn (19221996): Estructura de las revoluciones científicas (1962), se dio un cambio en la perspectiva y se empezaron a tener en cuenta los aspectos históricos, sociológicos y culturales de la ciencia.

\footnotetext{
${ }^{4}$ Fue un movimiento neopositivista que seguía algunas de las ideas planteadas por Comte. Dentro de los filósofos que sobresalieron de este grupo se encuentran: Morizt Schlick, Otto, Neurth, Hans Hahnn y R. Carnap.

5 Es claro que la investigación toma como punto de partida los problemas. Para investigar hay que plantear hipótesis que sirvan como intentos de solución. Una vez formuladas hay que comprobar dichas hipótesis y extraer consecuencias para ver si se cumplen o no. Si se cumplen confirman la teoría, y si no se cumplen la desmienten o falsean. Así, para una teoría sea aceptada como verdad científica tiene que poderse refutar, es decir, permitir su falsación a través de los hechos.

${ }^{6}$ Llama la atención acerca de la imposibilidad de que haya un método capaz de garantizar el desarrollo del pensamiento científico. Por el contrario, cada vez que avanza una ciencia se violenta el método utilizado y se descuida toso tipo de reglas metodológicas (Suarez, 2003). Y Asensi-Artiga y Parra-Pujante (2002), indica que, por diversas razones, la historia de la ciencia se convirtió para Feyerabend en un ingrediente esencial de la filosofía de la ciencia. Frente a Popper creía que no hay modo de delimitar, o demarcar - en terminología popperiana- la ciencia de lo que no lo es.

${ }^{7}$ Intentó adaptar el sistema de Popper (el Falsacionismo) a la nueva situación creada por Kuhn (Revolución Científica). $\mathrm{Su}$ intención era realizar una reconstrucción racional de la historia de la ciencia, mostrando que ésta progresaba de modo racional. La historia de la ciencia muestra que ésta no avanza sólo falseando teorías con hechos, hay que tener en cuenta la competencia entre teorías y la confirmación de teorías. Por ello sustituye el falsacionismo ingenuo de Popper por un falsacionismo sofisticado. En la realidad la ciencia no evalúa una teoría aislada, sino un conjunto de ellas que conforman lo que Lakatos llama programa de investigación científica.
} 
Según Kuhn la estructura de las revoluciones científicas se puede clasificar de descriptiva. Apenas dedica espacio a conceptos como verdad o conocimiento, $\mathrm{y}$ presenta la ciencia bajo un enfoque histórico y sociológico. Para Thomas Kuhn, la comunidad científica es la que permite el avance de la ciencia a través de teorías, "llamadas paradigmas o conquistas científicas universalmente aceptadas que durante un tiempo determinado brindan un modelo de problemas y soluciones aceptables para aquellos que trabajan en un campo de investigación" (Suarez, 2003; Cifuentes, 2016, p.69).

En perspectiva de Asensi-Artiga y $\mathrm{Pa}$ rra-Pujante (2002), las teorías dominantes bajo las que trabajan los científicos conforman lo que Kuhn ${ }^{8}$ llama paradigma. La ciencia normal es el estado habitual de la ciencia, el científico no busca criticar el paradigma, sino que da éste por asumido y busca la ampliación del mismo. Y señala que:

Kuhn utilizó la palabra paradigma por primera vez en 1959, en una conferencia sobre 'la tensión esencial', es decir, sobre los aspectos convergentes y divergentes de la empresa científica, que garantizan, respectivamente, la coherencia de lo que luego llamaría sus períodos de ciencia normal y los cambios conceptuales radicales que más tarde caracterizaría como revoluciones científicas.

\footnotetext{
${ }^{8}$ Se encuentra con una concepción de la ciencia como una actividad completamente racional y controlada (representada en aquellos años por el círculo de Viena). Kuhn, por el contrario, entiende la ciencia como una actividad concreta que se ha venido dando a lo largo de los siglos y que en cada época histórica presenta peculiaridades y características propias. En La estructura de las revoluciones científicas (1962), Kuhn señala las siguientes fases en el desarrollo de la ciencia 1. Establecimiento de un paradigma 2. Ciencia normal 3. Crisis 4 . Revolución científica 5. Establecimiento de un nuevo paradigma. Kuhn define paradigma de la siguiente manera: "Considero a los paradigmas como realizaciones científicas universalmente reconocidas que, durante cierto tiempo, proporcionan modelos de problemas y soluciones a una comunidad científica"
}

Si el número o la importancia de problemas no resueltos dentro de un paradigma es muy grande, puede sobrevenir una crisis y cuestionarse la validez del paradigma. Entonces la ciencia pasa al estado de ciencia extraordinaria o ciencia revolucionaria en el que los científicos ensayan teorías nuevas. Si se acepta un nuevo paradigma que sustituya al antiguo se ha producido una revolución científica.

En este sentido, Padrón (2007) sostiene que "nuevas epistemologías, no es en absoluto una expresión técnica, sino divulgativa, tal vez un tanto retórica, probablemente nacida más de los deseos de impactar acerca de novedades paradigmáticas supuestamente revolucionarias que de la necesidad de análisis críticos" (p. 13). Desde la década de los 90, aproximadamente, hasta hoy en día, dentro de los enfoques racionalista-idealista y empiristaidealista se han perfilado algunas nuevas epistemologías que constituyen el desarrollo de ciertos rasgos temáticoproblemáticos presentes desde las épocas anteriores.

En proceso de consolidación de las tendencias epistemológicas recientes, en dirección a la disertación expuesta por Padrón (2007), permite evidenciar que a partir de la década del 90 se comienza a desarrollar las epistemologías subjetivistas (racionalismo y empirismo idealistas) y que corresponden a: Contextualista, feminista, Social, entre otras; también las empiristas realistas: testimonial, Probabilística o bayesiana y Epistemología de la percepción. Estas tendencias de seguro contribuyen al desarrollo del pensamiento filosófico y científico y avanzan con el camino de la generación de conocimiento en las diferentes ciencias. 


\section{Conclusiones}

Cuestión importante, no solo estudiar la diversificación científica, sino que también se analice el origen mismo de la ciencia ya que es una respuesta a la inquietud gnoseológica propia del interés humano que estudia la naturaleza, el origen y el alcance del conocimiento. Una premisa valida en la actualidad más aun cuando se asiste a la globalización del conocimiento y cuando por causa de la tecnología se infiere una pseudo-adquisición del saber. Razón por la cual adquiere un carácter de imperativo el discernimiento sobre elementos de bondad o maldad, importancia o futilidad, entre otros antagonismos que refuerzan el proceso de enseñanza aprendizaje.

La filosofía de la ciencia, que se ocupa del investigar el conocimiento científico y la practica científica, al igual que de hacer seguimiento a al desarrollo y evolución de las teorías científicas y se apoya en diversos de sus tratados como la Epistemología, la cosmología, la lógica, la ontología y la teoría del conocimiento entre otros a fin de consolidar las bases filosóficas a fin de consolidar la validez y veracidad del conocimiento científico y de las teorías científicas.

El método científico, como camino de investigación científica y la confrontación de la ciencia. Sus dos principales métodos: deductivo e inductivo y las diversas etapas o fases permiten consolidar el mismo como fuente de la consolidación científica. Entre tanto que Karl Popper, conlleva a confrontar la falsación versus la verificación de la ciencia necesaria para un proceso científico; Thomas Kuhn, que propone las teorías llamadas paradigmas y las estructuras de las revoluciones científicas y desde luego Paul Feyerabend que se opone a un método eficaz para el desarrollo científico.
Para hablar de una filosofía de la ciencia no basta con tener una visión panorámica de lo que es filosofía y de lo que es ciencia. Tampoco es suficiente el seguimiento histórico de las opiniones y conceptos emitidos por los pensadores del pasado. Es necesario ubicarse en el pensamiento actual de los científicos más avanzados y respetar sus conceptos sobre lo que ellos consideran como ciencia, y es necesario entender que el dominio de la filosofía son los conceptos universales y abstractos que nunca pueden llegar a ser objeto de la ciencia.

Los afluentes que condujeron al rio de la afirmación: "el pensamiento filosófico $y$ científico han contribuido en la generación de conocimiento a lo largo de la historia y se consolida en el siglo XX y XXI". Permitieron fortalecer una salida al problema planteado, desde la argumentación de intelectuales que han desarrollado la investigación necesaria y en consonancia con la construcción de principios validos en relación a Filosofía de la Ciencia, la nueva Filosofía de la Ciencia, el Método como camino de validación del conocimiento y las tendencias epistemológicas recientes y contemporáneas en medio de un proceso sistemático y progresivo del desarrollo del pensamiento filosófico y científico conducentes al desarrollo del conocimiento.

\section{Referencias}

Azcárraga, J. A. (2002). Ciencia y Filosofía. Mètode, Anuario, (2002), 40-47. Asensi-Artiga, V. y Parra-Pujante, A. (2002). El método científico y la nueva filosofía de la ciencia. Anales de documentación, 5(1), 9-19.

Bojaca, A. J. (2000). ZYX La lengua filosófica Universal. Colombia: Edición A\&C Diseñadores Ltda. 
Carman, C. (2007). La filosofía de la ciencia en el siglo $X X$. Recuperado de: https://ubc.edu.mx/plataformavirtual/mod/folder/view.php?id=1631.

Iranzo, V. (2005).Filosofía de la ciencia e historia de la ciencia. Quaderns de filosofia i ciència, 35, pp. 19-43. Universitat de València

Cifuentes-Medina, J. E. (2016). El método científico y la nueva filosofía de la ciencia: aportes y perspectivas. Rastros Rostros, 18(33), 61-70. Recuperado de http://dx.doi.org/10.16925/ ra.v18i33.1681

Cifuentes, J. y Pedraza, J. (2016). Teorías no euclidianas y Filosofía de la Ciencia siglo XX: tendencias epistemológicas siglo XXI. Revista Criterios, 23(1), 211-229.

Gandía, C. E. (2004). Filosofía de la Ciencia. Recuperado de: https://ubc. edu. $\mathrm{mx} / \mathrm{plataformavirtual} / \mathrm{mod} / \mathrm{fol}$ der/view.php?id=1631

González, A.A. (1969). Historia de la Filosofía: En cuadros esquemáticos. $6^{\text {a }}$ ed. Madrid: Ediciones EPESA S. A.

Giraldo, J. (2014). ¿Cuál es el lugar del sujeto en la educación virtual?. Cultura, Educación y Sociedad, 5(1), 69-89. Recuperado de: http://revistascientificas.cuc.edu.co/index.php/ culturaeducacionysociedad/article/ view/996/pdf_213.

Kuhn, T. (1975). La Estructura de las Revoluciones Científicas. México, D.F.: FCE.

Lakatos, I. (1982). La historia de la ciencia y sus reconstrucciones racionales. $2^{a}$ ed. Madrid: Tecnos.
Ortiz, F. y García M. (2010) Metodología de la investigación: El proceso y sus técnicas. México, D.F.: Limusa

Padrón, J. (2007). Tendencias epistemológicas de la investigación científica en el siglo XXI. Cinta de Moebio, 28(1), 1-28. Recuperado de: http://www. moebio.uchile.cl/28/padron.html.

Senior, J. (2001). El surgimiento de las teorías no euclidianas y su influencia en la filosofía de la ciencia del siglo XX. Revista Colombiana de Filosofía de la Ciencia, 2(4), 45-63.

Rivadulla, A. (2004). La filosofía de la ciencia hoy: problemas y posiciones. Perspectivas del pensamiento contemporáneo / coord. Por Juan Manuel Navarro Cordón, Vol. 2, 2004, ISBN 84-9756-231-3, págs. 109-163Universidad Complutense de Madrid.

Suarez, M.G. (2003). Pienso... Filosofía y su Historia II. $1^{\text {a }}$ ed. Bogotá, D.C.: Editorial Voluntad.

Martínez, J. E. (2001). El surgimiento de las teorías no euclidianas y su influencia en la filosofía de la ciencia del siglo XX. Revista colombiana de filosofía de la ciencia, 2(5), 45-63.

Medina, J. E. C. y Suaréz, J. I. P. (2017). Teorías no euclidianas y Filosofía de la Ciencia siglo XX: tendencias epistemológicas siglo XXI. Revista Criterios, 23(1), 211-229.

Verano, G. L. (2004). Pienso... Filosofía y su Historia I. $1^{a}$ ed. Bogotá, D.C.: Editorial Voluntad.

Valdés, O. J. (2015). Filosofía de la Ciencia. (Documento de trabajo académico). 